ORAL AND FACIAL DEFORMITY

By C. Kerr McNeil, Ph.D., L.D.S. Pp. I27, with I9I illustrations. London: Sir Isaac Pitman \& Sons, Ltd. 1954. $25 \mathrm{~s}$.

The number of medical and dental practitioners familiar with Dr. McNeil's work is strictly limited and some regret must be expressed that this book, excellent as it is, in some respects fails to tell the full story. It is a fault on the right side that some workers hesitate to rush into print at too early a stage, but Dr. McNeil admits himself that his researches are very much more advanced than this book would indicate. The book is not much more than his thesis for his Ph.D., and whilst that in itself was more than worth while reading some disappointment will be felt that the work being done at the Glasgow Dental Hospital is not being made more widely known. The technique for closing palatal defects by stimulation of growth of tissues is one that ought to be studied and followed over a far greater field than one academic centre, and even if the dental profession is becoming gradually familiar with this technique it should be made more readily available to a wider audience.

There can be no doubt that repair of acquired or congenital injuries of the face and jaws is the work of a team consisting of a plastic surgeon, a dental specialist and perhaps a speech therapist and it is Dr. McNeil's experience in the late war that led him to proceed after demobilization to the study of congenital malformations. He was fortunate in becoming a member of a good team and has been able to formulate original ideas on the aetiology of congenital malformations, the stimulation of tissue growth and the incidence of twinning where there is an occurrence of congenital oral deformities.

All those concerned with this important branch of surgery will want to hear further from this research of Dr. McNeil and what he has told us now cannot but stimulate interest in what is obviously a new approach to the subject. The incidence of malformations of the face and jaws does not decrease and indeed the cases brought for early treatment increase so that all new knowledge is eagerly sought. Whilst a welcome is extended to Dr. McNeil's book it is earnestly hoped that we shall hear more from this source in the near future. Primarily written for the dental specialist there is a great deal in the book for the plastic surgeon who will want to keep up to date on what can be done to further his work after the immediate operative stage.

S.R.

\section{MEDICAL ELECTRONICS}

By G. E. Donovan, M.D., M.Sc., D.P.H. Pp. $x+215$ pages, with 37 illustrations, one in colour. London: Butterworth \& Co., Ltd. 1953. 30s. Electronics deals with thermionic valves, cathode ray tubes, $\mathrm{X}$-ray tubes and other devices in which electrons move through an evacuated or gas-filled space. In giving an account of electronics in medicine, the author of this book has wisely refrained from trying to explain the working of electrical circuits. Anyone who wants a reaß̋ understanding of electronics must build his own cir- 3 cuits and he will find that there are many good books to help him. What this work gives is a concise re-c. view, with references, of the hundreds of differen pieces of electronic apparatus which have been des cribed in the literature of medical practice ando research. For completeness, the Einthoven stringo galvanometer and some other non-electronic instruments have been included.

The book has the fault of being too concise in places and the busy doctor, mentioned in the pre-w face, may have to consult his colleague, the hospitalphysicist, on a number of points. For instance, the triode X-ray tube is mentioned, but no indication is given of how it differs in construction, properties oro uses from the ordinary $\mathrm{X}$-ray tube. The reviewer was irritated by the repeated use of variations on the phrase 'it should be valuable in research' and by such expressions as 'the thread of amplificationo runs through the fabric of medical electronics,' but these are minor points which merely spoil the pleasure of reading. Otherwise this is an excellento and long-needed book and it will prove invaluable to all who use or who contemplate using electricap apparatus in medical work.

\section{S.R.}

\section{Ciba Foundation Symposium}

THE CHEMICAL STRUCTURE OF PROTEIR̂: Edited by G. E. W. WolstenhOlME, O.B.E.; M.A., M.B., B.Ch. Pp. xii +222 , with क्qे illustrations. London: J. \& A. Churchill LEd. 1953. 25 s.

This report of a symposium held by the Ciban Foundation contains 16 short reports on someD aspects of protein chemistry which are mainly concerned with chemical degradations and the charac-o terization of the proteins and their fragments.

Not many years ago the determination of protein structure seemed a hopeless problem but reading. this book gives one the impression that the solution is in sight, even if some way off. The techniques are available and, for the smaller proteins at anyo rate, settling the structure only seems a matter of time and labour.

This is partly the result of the micro-techniques of partition and ion exchange chromatography and micro-electrophoresis which are dealt with in their application to particular cases by several of the con $\rightarrow$ tributors. In particular the extension of partition chromatography to the proteins themselves hasi given a new method of checking purity and has shown that several crystalline proteins contain morev than one component.

Many of the contributions deal with ingenious chemical methods of partial hydrolysis and markinge the ends of polypeptide chains. In theory, at anf rate, it appears that with some of these the structure of any polypeptide can be established by nibbling? away amino acids one at a time from the end of the chain.

J.N.S. 